\title{
Probing elastic properties of soft materials with AFM: data analysis for different tip geometries
}

Marius Chyasnavichyus, Seth L. Young, Ren Geryak, and Vladimir V. Tsukruk*

School of Materials Science and Engineering, Georgia Institute of Technology, Atlanta, Georgia, 30332, United States

\section{Abstract}

It has become increasingly common to use atomic force microscopy measurements to probe mechanical properties at the nano-micro level. The data obtained from these measurements, however, must be subjected to specific models for deconvolution of the effect of the probe's tip size and shape. While analytical models have been developed to assist in this endeavor, a thorough understanding of the limits of these models is essential to fitting data accurately. In this report, we explore the relationship between three different analytical tip shape models for the AFM tip (spherical, parabolic, and conical indenters) and present an analysis of mechanical testing on selected materials. Along with this, we present a simple numerical method for computing the contact radius for true spherical contact. The role of tip size (large vs small radius) on the limitations of data analysis and the benefits and drawbacks inherent to different tip sizes is discussed. Our analysis demonstrates the ability to accurately apply multiple models to a given data set, while also showing the limitations of simple analytical models to accurately describe tip-sample interactions outside of certain indentation regimes.

Keywords: contact mechanics, surface force spectroscopy, soft materials, data analysis

*To whom correspondence should be addressed: vladimir@mse.gatech.edu 


\section{Introduction}

The quantitative characterization of surface microstructure, morphology, and properties of soft materials such as polymers, proteins, hydrogels, biomacromolecules and corresponding bionanocomposites is critical for many areas of industry, defense, and medicine $[1,2,3]$. As is known, atomic force microscopy (AFM) methods provide variety of tools to access these properties by measuring the interactions of a sharp probe with a specimen surface, and can often produce topographical images with near atomic resolution [4]. Furthermore, unmatched force sensitivity allows one to non-destructively investigate a variety of materials ranging from very compliant live cells to extremely stiff cellulose fibrils and rigid polymers $[5,6,7,8,9,10,11,12]$. The versatility of AFM facilitates the measurement of surface forces in controlled environments, as well as with a variety of tip shapes and cantilever spring constants [13,14]. These methods have been successfully employed in our laboratory to study the micromechanical behavior of thin and ultra-thin polymer films [1], silicone-based contact lenses [15], biological sensing systems [16], and cell encapsulation membranes [17] to name a few. However, experimental routines as well as data analysis for the consistent extraction of accurate, quantitative data is not always a straight forward process.

Surface micromechanical properties calculations involve specific contact mechanics models which closely resemble actual tip-sample contact geometry. It is known that even for the simplest cases of semi-infinite elastic half-spaces and axisymmetric punch indentations, the calculated elastic modulus will strongly vary with the size of the probe and punch profile shape. Often this geometry is not precisely known, and as a result the standard Sneddon's model for a paraboloid indenter on a flat half space may be improperly applied as opposed to say a conical, or spherical indenter. Furthermore, it is vitally important to precisely monitor and control sample deformation. This is especially true for very soft material surfaces where the nature of the tip-sample contact geometry can change due to increasing penetration, or in the case of a thin film on top of a the stiff substrate which could increase apparent modulus. In the case of compliant cells, or other soft materials with irregular shapes, increased forces may lead to structural 
deformations instead of the local ones, which will yield structural rigidity instead of the localized mechanical properties the investigator may be seeking.

In this article we will discuss several aspects of measurements of elastic properties of materials with AFM. We will examine effects of tip shape variation on FDC fitting and elastic modulus extraction as well as importance of selection of the particular tip shape model for elastic modulus measurements. In particular, we will discuss parabolic, spherical, and conical models and will examine specific cases where these models should be used. In addition we will introduce improved algorithm for fast fitting of FDCs for spherical tip shape approximation, as spherical model is the only model, where there is no analytical equation for tip-surface contact area dependence on penetration. Finally, we discuss how probe size affects results of FDC fitting by using as example soft synthetic and biological materials in dried and swollen states. For that purpose we used silicon probes annealed at high temperature to achieve higher tip curvature. All of the analysis procedures have been incorporated into an offline FDC analysis program, Micromechanical Analysis (MMA) Software, introduced in our lab.

\section{Experimental}

2.1 Materials. Regenerated silk fibroin was obtained via extraction from Bombyx mori cocoons, as described previously $[18,19,20]$. The silk ionomers were used as described in a previous report [21]. These biopolymers were made into films through spinassisted layer-by-layer ( $\mathrm{LbL}$ ) deposition by alternating casting of aqueous solutions of the polycationic and polyanionic silks onto polystyrene coated silicon wafers.

In this study we also use examples from a commercially available soft polymeric contact lens, Balafilcon A (Purevision, Bausch \& Lomb Inc.). The lens was measured immediately after removal from the original blister pack containing saline solution and discarded after one day of measurements. The lens was sectioned as described in detail in an earlier study in order to create a flat region conducive to AFM measurements [15]. A small droplet of the saline solution taken from the blister pack 
was placed at the scanning surface of the lens and measurements with the AFM were made inside of this droplet.

For the example of harder polymeric material we use the polystyrene (PS, weightaverage molecular weight $\sim 250,000 \mathrm{~g} / \mathrm{mol}$, Sigma-Aldrich), which is a well-known amorphous polymeric material with values of elastic modulus frequently reported in the literature to be around $3 \mathrm{GPa}$. A saturated PS solution $(150 \mathrm{mg} / \mathrm{mL})$ in chloroform was cast in a glass Petri dish and held under vacuum for several days to remove solvent. The final thickness of this substrate was on the order of 1-2 mm. AFM measurements were made directly on the top surface of the PS substrate without removing from the glass Petri dish inside a deionized water droplet.

2.2 Atomic force microscopy. All AFM measurements were performed using a Dimension Icon microscope (Bruker) equipped with a Nanoscope $V$ controller. Rectangular n-type Si probe chips containing four cantilevers each, with nominal spring constants ranging from of $0.2 \mathrm{~N} / \mathrm{m}$ to $40 \mathrm{~N} / \mathrm{m}$, were obtained from MicroMasch (Lady's Island, South Carolina). For some experiments, we utilized probes with moderatelysized tip radii ( 200-500 $\mathrm{nm}$ radius), which were created by annealing as received AFM tips at $1100^{\circ} \mathrm{C}$ in air for $6-12$ hours.

The radius of curvature at the tip apex was estimated prior to each experiment by scanning a standard titania roughness sample provided by Bruker and performing a blind estimation algorithm in order to make a 3D reconstruction of the tip shape [22]. Several cross sectional profiles $(>5)$ across the tip apex were taken from the reconstructed tip image to be analyzed in our MMA Software to determine tip radius of curvature. The radius of curvature was taken as the average from the set of section profiles. In the case of the moderately-sized probes, tip shape was also independently investigated by using by collecting SEM images on a Hitachi S-3400N thermal emission SEM. AFM tips were placed in a spring loaded clamp and scanned in ultra-high vacuum at magnifications of $\sim 25 \mathrm{k}$ and $\sim 100 \mathrm{k}$ with an accelerating voltage of $5 \mathrm{kV}$.

Scanner deflection sensitivity was determined for each cantilever prior to any measurements by collecting force distance curves (FDCs) on a sapphire crystal. The 
cantilever spring constant, $k$, was measured using the thermal tune method [23,24]. Topography images were collected on the sample surface prior to taking FDCs (to check for surface uniformity) in peak force tapping mode using the established procedure [20] where the AFM tip is moved sinusoidally in the z-direction (normal to the surface which is being measured) and a user defined force (typically a few $\mathrm{nN}$ ) is used as a feedback in order to track the surface topography. Scan areas ranged from 500 $\mathrm{nm} \times 500 \mathrm{~nm}$ to $20 \mu \mathrm{m} \times 20 \mu \mathrm{m}$ using resolutions of either $512 \times 512$ pixels or $1024 \times$ 1024 pixels. Scan rates were chosen within $0.5-1 \mathrm{~Hz}$.

For elastic modulus calculations it is extremely important to precisely determine the initial contact point where the tip starts to penetrate the sample. In these studies, this point is calculated automatically using an algorithm, described in detail elsewhere [25]. In short, this is a "double alarm" algorithm where the program follows the deflection of the approach curve. When the deflection reaches certain threshold deviation from the baseline deflection, an alarm of the first kind is set for this point. When a similar deflection deviation is reached further down the approach curve the first alarm is erased and alarm marker is attached to the new point. When a deviation of deflection above the certain set value is reached, an alarm of the second kind is issued. The algorithm then goes back to the point where the alarm of the first kind was issued and this point is assigned as the first contact point. For baseline-corrected curves with low background noise, the aforementioned algorithm was found to accurately find the first point of the onset of deflection.

\section{Discussion}

\subsection{Model of the tip sample contact.}

The present measurements have been done under the assumption of the elastic behavior of the tip-sample contact. All force-distance curve measurements in this study have been performed in liquid. This was done to bring the experiments in line with materials working conditions for the case of silk ionomer layers and contact lens. At the 
same time measurements in fluid reduced surface energy and eliminated effect of the liquid bridge formation, that can introduce significant snap in deflection changes not related to the adhesive properties of the material. For most of the measurements performed in this study deflections below baseline were nonexistent. In the case of presence of negative deflections in retract portions of the FDC the negative deflections were less than the $5 \%$ of the positive deflection used for the modulus calculations,

suggesting negligibly small adhesion forces ${ }^{26}$. Therefore for all calculations in this study we have assumed non adhesive behavior. For the case where adhesion behavior becomes significant, one should use more elaborate models [27]. These models are more complex for practical applications were shown to work well for the case of parabolic tip shapes or spherical tip shape in the case of indentations small compare to the radius of curvature of the tip.

The model described in this manuscript does not involve any discussion of timedependent effects here as we have devoted a separate recent article to full viscoelastic analysis of soft materials [28]. In addition, we assumed no plastic deformation of the sample at the penetration depths used in this study. Sample surface examination after the indentation measurements in all the cases revealed no residual deformation of the sample surface. It should be noted that at much larger penetrations plastic deformation does occur and the models presented in this study fail to describe mechanic properties in this case. Discussion of the FDCs with the plastid deformation region can be found in recent literature [29].

\subsection{Elastic modulus calculation}

As known, the contact mechanics equation for the elastic modulus calculation developed by Hertz fits well only for indentations much smaller than the radius of the curvature of the indenter and thus generally not applicable to most of practical conditions found for soft materials. On the other hand, it was shown by Sneddon [30] that elastic modulus can be found for the case of larger indentation of rigid axisymmetric punch with known profile into the elastic half space. 
The its general form the solution of Sneddon's model requires solving the system of nonlinear equations with numerical integration along with the functional representation of the tip profile, which makes it very compute-intensive. Recently a method of piecewise integration was presented [31], which allows calculation of elastic modulus for the known tip profile using linear interpolation of the tip profile taken from SEM image of the tip. This method was shown to have high precision of the modulus calculation, however it still requires considerable amount of calculations and uses tip profile taken along a single direction across the tip.

Here we suggest using several simple tip shapes: cone, paraboloid and sphere, which have simplified equations for elastic modulus calculations. Out of three models only for the spherical tip shape, there is no analytical solution and some calculations are required. However for this case we have found a unique precalculated set of initial values, which allow the algorithm to converge in just several iterations. Presented method uses several sections of tip profile, providing averaged values, which describe the tip shape. For all tip shapes the elastic modulus is calculated through simple linear regression, which helps to evaluate the validity of the applied models: if the strong nonlinear deviations occur then the more precise calculations, for example through above mentioned piecewise integration are required, or the tip-sample interaction deviated from simple elastic behavior.

\subsection{Spherical versus parabolic tip shape.}

It was shown by Sneddon [30] that there is a simple analytical solution for cases of large indentation which inversely relates the elastic modulus to $\delta^{3 / 2}$ for a rigid parabolic indenter, according to eq. (1):

$$
F=\frac{4 \sqrt{R}}{3} \frac{E}{1-v^{2}} \delta^{3 / 2}
$$


where $F$ is the applied force, $R$ is the effective tip-sample radius of curvature, and $v$ is the samples Poisson's ratio. From this it the penetration depth can also be related to the radius of the contact area circle, $a$, by eq. (2):

$$
\delta=\sqrt{a R}
$$

For the case of truly spherical contact, these equations become somewhat more complicated as there is no analytical solution which relates applied force to penetration depth. In this case the applied force is related to the contact area radius via eq. (3):

$$
F=\frac{E}{1-v^{2}}\left[\left(a^{2}+R^{2}\right) \ln \left(\frac{R+a}{R-a}\right)-2 a R\right]
$$

while the penetration depth is separately related to the contact area radius by additional eq. (4):

$$
\delta=\frac{a}{2} \ln \left(\frac{R+a}{R-a}\right)
$$

This difference in the contact behavior applicability is related to the fact that the contact area for the spherical indenter is finite and cannot exceed the radius of the indenter, which can be the case for some AFM probes (for example, see Fig. 1A, B), whereas for the parabolic indenter this area increases indefinitely and is proportional to the square root of penetration. Fig. $1 \mathrm{C}$ shows penetration depth versus contact area radius both normalized to indenter radius, which allows for the examination of the difference in the contact area between the two models. Although for a wide variety of hard materials large penetrations will cause plastic deformations and cannot be fitted with the elastic model [28]. However, for many soft polymeric materials large penetrations ( $\delta$ on the order of several $R$ ) are easily achieved in the elastic regime above glass transition [28].

For simplicity of comparison of the two models in the following discussion, we will assume the radius of the spherical punch, $R$, to be the same as the radius of curvature of the apex of a paraboloidal punch.

Under these conditions, the initial regions of the penetration curve for both models will be exactly the same (See Fig. 1C). This is a consequence of eq.(2) being the limiting 
case of eq. (3) for $a \ll R$. However, there is a large discrepancy between parabolic and spherical model in the terms of contact area for larger penetrations. It is $\sim 9 \%$ for $\delta=0.5 R$ and grows to $\sim 20 \%$ at $\delta=1.0 R$ (See Fig. 1C). Therefore under relatively large indentation depths incorrect selection of the fitting model can result in substantial errors in the calculated elastic modulus value. This is especially true for the case for the tips with larger radius and AFM tips, which each have a unique shape (Fig. 1A).

If one would try to approximate a spherical indenter with a parabolic profile by curve fitting one would find that the radius of curvature at the apex of the parabola would be smaller than $R$, thus increasing discrepancy between the two models even more than discussed above. As an example, we have assumed a dimensionless perfect sphere with a radius of $R=1$ (Fig. 1B). Fitting this profile with the method of the least squares using points up to $0.7 R$ with the general formula for a parabola, $y=a x^{2}+b$ gives the values $a=0.7491$ and $b=-0.0334$, which gives the curvature of the apex $R_{\text {parabolic }}=$ $\frac{1}{2 a}=0.6675$. In this case the curves of $\delta / R$ versus $a / R$ are different than those shown previously (see Fig. 1D), with 22\% discrepancy at $\delta=0.5 R$ and just $2 \%$ at $\delta=1 R$. But in both cases error dramatically grows beyond the threshold: $\delta \cong 1.5 R$. Both examples show that the simple analytical solution in eq.(1) cannot be used for the tips with the spherical profile ant larger penetrations.

In terms of elastic modulus calculation the main difference between the parabolic and spherical model is absence of an analytical equation which directly relates force and penetration for spherical tip shape. This is in contrast to the parabolic and conical models (discussed below), where force is proportional to the penetration through the power law and the elastic modulus is calculated through the constant of proportionality. In order to calculate modulus using a truly spherical indenter, it is thus required to first calculate contact area corresponding to each penetration in the experiment through the eq. (4) and then use eq. (3) to calculate the modulus. Since eq. (3) is nonlinear and does not have an analytical solution, solving for $a$ can take considerable amounts of time using simple minimization algorithms, especially for gathering statistics for multiple curves with high numbers of data points, which is not very practical solution. 
Another problem of the simple minimization algorithm is the precision in the contact area calculation for high penetration values (see Fig. 1C). One can see that, when $a / R$ approaches 1 , large changes in penetration produce only minute changes in the contact area.

In order to get around this complication, we have developed fast calculation algorithm, which allows for fast real-time calculations of elastic modulus. The algorithm exploits the monotonic behavior of eq. (3) as well as the fact that the function $a(\delta)$ has limit at $a=R$. We have pre-calculated a set of the initial guess values, that depend only on $\delta_{\max } / R$, where $\delta_{\max }$ is the maximum penetration observed in the experiment. Then, eq. (4) can be solved for $a$ in just a several iterations by using Newton-Raphson method with precision of higher than $0.01 \%$ for each value of $a$. When the values of $a$ are known for each value of penetration, eq.(3) is used to find elastic modulus through simple linear regression analysis. The method is described in detail in Supporting Information.

As a practical example, Figure 2 shows FDCs taken on swollen soft polymer contact lens. Figure $2 \mathrm{~A}$ shows $\delta^{3 / 2}$ versus $F$ fit with Sneddon's model for a rigid parabolic punch. From the plot, one can see that that there is a significant deviation from the linear behavior for $\delta^{3 / 2}$ vs. $F$. The calculated elastic modulus, $1.75 \pm 0.22 \mathrm{MPa}$ (here and onward data is presented value \pm STD, statistics gathered from 100 curves), can be used to superimpose theoretical FDC onto the experiment, which shows different, steeper characteristic FDC shape (Fig. 2B). Figure 2C shows a plot of $\left(a^{2}+R^{2}\right) \ln \left(\frac{R+a}{R-a}\right)-2 a R$ (denoted as $\delta$ - $a$, with units of $\mathrm{nm}^{2}$ ) versus $F$ (the linear dependence shown in the eq.(3)), which has significantly lower fitting error than the parabolic model, with calculated elastic modulus of $2.83 \pm 0.24 \mathrm{MPa}$. As can be seen in Fig. 2D, the same superimposition results in a much closer fit over the entire FDC.

As mentioned, in the initial stages of indentation the parabolic and spherical models behave similarly. Insets in Fig. 2A and Fig. 2B are the initial portions of the FDCs also fitted with Sneddon's model for a rigid parabolic punch, giving 2.79 $\pm 0.15 \mathrm{MPa}$. From this it can be seen that the parabolic model fits very closely with the experimental data with minimal deviation. 
To summarize, Sneddon's model for a rigid parabolic punch and spherical punch gives very similar results for small indentation depths, with the calculation for the parabolic tip shape being much less cumbersome. As a result, for many users the parabolic model is employed (either knowingly or unknowingly) in preference to the truly spherical model. However, as can be seen in Fig. 1A and Fig. 2C, the shape of AFM tips is not always exactly parabolic, and can be actually more close to a more cylindrical punch capped with a hemisphere. For such cases we have developed a simple algorithm for numerically fitting our data with the spherical equations, and have demonstrated a case in which it is preferential to use this model for more accurate values of elastic modulus.

\subsection{Conical versus parabolic tip shape.}

For very compliant soft polymeric and biological materials, especially in the case when they are in the highly swollen state, very high deformations can be achieved without inducing plastic deformation. Such large indentations require fitting with contact mechanics model which considers a rigid conical indenter on a semi-infinite half-space. During measurements involving large penetration depths, the initial parabolic shape of the tip makes very small contribution to the overall contact area increase, thus the tip can be considered conical in shape. Fig. 3 shows an indentation experiment performed on silk ionomer layers of $140 \mathrm{~nm}$ thick. The parabolic model can be used only for the small initial portion $(<20 \mathrm{~nm})$ where contact force changes as $\delta^{3 / 2}$, after which the penetration changes as $\delta^{2}$, signifying a different indentation regime.

For the conical tip Sneddon calculations give the following equations [30]:

$$
\begin{gathered}
F=\frac{2}{\pi} \frac{E}{1-v^{2}} \tan (\alpha) \delta^{2} \\
\delta=\frac{1}{2} \pi \cdot a \cdot \tan (\alpha)
\end{gathered}
$$

It can be seen that for the conical model the contact area a grows linearly with penetration. For conical tip shape, the determination of tip shape can be done through 
either electron microscopy [32] or blind estimation [33]. While possible to obtain an accurate reconstruction down to a few $\mathrm{nm}$, obtaining an accurate estimate of tip opening angle generally requires less resolution than determining the tip radius at the apex, which allows for better determination of the tip-sample interaction. Additionally, tip damage or contamination of the tip apex does not generally impact the behavior of a conical tip at sufficiently deep penetrations (since the defect might contribute to only a fraction of the penetration behavior). In the geometric analysis performed by Berla et al. [34] a real tip can be modeled as a combination of a conical tip with a parabolic terminus defect (often called a "blunted tip"). The projected conical tip will overshoot the real end of the tip by an amount found to be $h=k R m^{2}$, where $k$ is a constant specific to this equation determined by Berla et al., $R$ is the radius of curvature of the AFM probe, $m$ is the slope of the line parallel to the side angle of the AFM tip.

By fitting the profile of the tip apex with a parabolic function, we determined that our probe tip had a radius of about $7 \mathrm{~nm}$, which corresponds well with the manufacturer's stated estimate. The conical profile of the tip was obtained by taking a linear fit of the reconstructed tip profile further away from the tip apex (roughly 15-20 nm away) and resulted in a cone semi-angle of $25.3^{\circ}$. In our case, this leads to projected cone height which extends roughly $26 \mathrm{~nm}$ past the real tip. In our analysis, we compare the results for a parabolic tip, which is fit to small penetrations $(0-20 \mathrm{~nm})$, and a conical tip, which is fit to deeper penetrations $(15-50 \mathrm{~nm})$ and has its starting point adjusted to account for the $26 \mathrm{~nm}$ of the "phantom" tip height.

Because we do not correct for the effects of the blunted tip, the region of small penetration is not accurately fit by this model. As a result, a significant number of experimental points past this initial region is required to obtain a suitable fit for our conical model. In some materials, this constraint coupled with the sharp tips used in this study can be problematic because the large indentations can lead to a significant degree of plastic deformation in the sample [35]. With a swollen thin film of modified silk fibroin, we were able to achieve relatively large penetrations $(80 \mathrm{~nm})$ with a negligible amount of plastic deformation as indicated by a lack of hysteresis in the approach and retract curves, and no changes in sample topography after indentation experiments. 
For these films, we fit a parabolic indenter with radius of curvature of $7.09 \mathrm{~nm}$ and a conical indenter with an opening semi-angle of $25.3^{\circ}$. In Fig. 3 , the fitting results are displayed. As can be seen in the two graphs, the obtained fitting is quite good for both models and the results obtained for the modulus calculation is comparable. By analyzing 25 of such force distance curves, we found that a modelling of the data based on paraboloid geometry gave an elastic modulus of $47.1 \pm 9.3 \mathrm{MPa}$, whereas modelling a conical geometry led to an elastic modulus of $52.7 \pm 5.2 \mathrm{MPa}$. Given the observed similarity of these values (within the experimental error), the approach used in this study seems to be valid. Of note, the conical model appears to exceed the parabolic model by an average of $15 \%$, though such a deviation is not statistically significant. Considering the relatively high degree of uncertainty in the parabolic fits, we suggest that our model suffers from a degree of uncertainty in the tip-sample interaction within the first few nanometers (particularly, a precise tip profile and starting point location). This can complicate the choice of the starting point adjustment needed to accommodate the conical model, and thus further investigation is warranted.

\subsection{Tip size variation.}

In many cases, the use of probes with large tip radius or colloidal beads can be advantageous over sharp probes. Foremost, it is known that larger tip radii reduce the maximum stress on the material in the area just blow the tip-sample contact point, and thus the use of probes with larger tip radii significantly reduces the possibility of inducing plastic deformation during FDC collection. Quick examination of the simple Sneddon model of a rigid paraboloidal punch shown in eq. (1) reveals that, for a given applied force $F$, penetration depth (as $\delta^{3 / 2}$ ) and tip radius of curvature (as $\sqrt{R}$ ) are inversely proportional. As a result, one can see that for extremely soft samples such as loosely networked hydrogels or other compliant biomaterials, increasing tip size can significantly reduce the penetration depth for a given applied force, thus diminishing the probability of inducing plastic deformation on the sample surface. Furthermore, because of this inverse proportionality, in cases where high lateral resolution is not required (e.g. thick, homogeneous material surface), much higher force resolution can be achieved with large radii probes because larger much larger forces can be applied to 
the surface while maintaining low penetration depths. This can serve to significantly reduce data analysis errors associated with few data points, and can also be used to overcome the snap-in instabilities associated with large probes as the snap-in forces becomes negligible compared to the overall applied force.

However, for many SFS experimental setups, the use of very sharp probes (tens of $\mathrm{nm}$ radius of curvature) is advantageous as it provides higher lateral resolution in surface mapping of properties facilitating the discernment of different phases of a heterogeneous material surface, or it can allow for the measurement of the micromechanical properties of small structures such as fibrils. We present two examples of high resolution mapping of surface mechanics (adhesive interactions and apparent modulus) in Fig. 5 to better illustrate this point.

In Fig. 4A-C the surface of regenerated silk fibroin macromolecules deposited on a silicon wafer is shown with the corresponding adhesion image taken in Bruker's PeakForce QNM mode. As can be seen, the silk fibroin macromolecules interact with the AFM tip differently than the silicon surface, resulting in distinctly different surface adhesion behavior. An AFM tip with a large radius would be far less discriminant between the two materials at the surface. Furthermore, larger radii tips are more prone to capillary forces due to the presence of a thin water layer at the surface, which would make crisp adhesion mapping shown in Fig. 4B nearly impossible.

As another example, Fig. 4D-F shows a buckled silk-ionomer film surface with the corresponding DMT Modulus (taken using PF-QNM). In this case, the raised buckled regions are generally between $45 \mathrm{~nm}$ and $90 \mathrm{~nm}$ and are not supported by the underlying substrate (Fig. 4F shows sectional profile). Consequently, these structures are extremely compliant when force is applied by an approaching AFM tip. Because a large radius probe would contact many of these high features first it would be extremely cumbersome to determine true contact area, resulting in high error in calculating the sample elastic modulus. Furthermore any calculated modulus for FDCs taken with a large radius probe on these samples would be a composite value of the extremely complaint buckles and the stiffer material. Scanning with a sharp probe allows for 
access to substrate-supported regions of the silk ionomer surface, and more accurate estimates of the surface mechanical properties (here the apparent DMT model gives 100-300 MPa for supported regions and close to $0 \mathrm{MPa}$ for buckled regions).

In order to demonstrate how the analysis with AFM probes of considerably different radii can influence results, we present here several micromechanical measurements taken on a thick glassy PS film made by casting a highly concentrated solution dissolved in chloroform. In one set of measurements FDCs were taken in fluid (Nanopure water) using as-received AFM tips ( $R=\sim 20 \mathrm{~nm}$, Fig. 5), while in another set of measurements thermally annealed tips were used $(R=\sim 330 \mathrm{~nm}$, Fig. 6). Even with well-known polymeric materials such as PS here, processing conditions can create the possibility for surface mechanical behavior which is not typically observed in bulk measurements. In the specific case of our thick-cast PS films, we consistently observed ultrathin surface layer with an abnormally low elastic modulus, which, we speculate, is contamination and low molar mass coatings.

Fig. 5 shows a representative FDC collected using a sharp probe $(k=6.9 \mathrm{~N} / \mathrm{m})$, with a total maximum penetration depth of approximately $8 \mathrm{~nm}$. Fitting the $\delta^{3 / 2}$ versus applied force data obtained from the FDC in Fig. 5A resulted in a calculated elastic modulus of $569 \mathrm{MPa}$. Examination of $>20 \mathrm{FDCs}$ from different locations along the surface resulted in an average modulus of $479 \pm 111 \mathrm{MPa}$.

When this same surface is probed using an annealed AFM tip with a large radius of curvature ( $\sim 330 \mathrm{~nm}$ tip radius) the FDC behavior is different. Fig. $6 \mathrm{~A}$ and $6 \mathrm{~B}$ show a representative FDC and $\delta^{3 / 2}$ versus applied force plots. In this case the applied force was adjusted by using a stiffer cantilever $(k=65.8 \mathrm{~N} / \mathrm{m})$ such that a similar penetration depth could be reached as compared to the FDCs taken with the small radius probe. In this case the maximum penetration depth was $\sim 7.5 \mathrm{~nm}$ (Fig. 6D has raw penetration depths determined from the experiment). As can be seen in Fig. 6B, there is some significant fitting error associated with using the parabolic model for these curves. This is a result of the apparent "layered" behavior seen in the plot of $\delta^{3 / 2}$ vs. F (Fig. 6B), as one linear slope transitions gradually to another, representing a change from a lower 
modulus to a higher modulus. Fitting the overall curve, therefore, represents a composite value of the two separate moduli, giving a calculated modulus of $1.3 \mathrm{GPa}$ in this example.

However, this curve was also analyzed in at different penetration depths. For low penetration depths (e.g. $\sim 4 \mathrm{~nm}$ seen in Fig. $6 \mathrm{C}$ ) the sample surface appears much more compliant with a modulus of approximately $650 \mathrm{MPa}$, which is much more closely associated with the value determined from FDC analysis with a sharp probe. If the region of the FDC corresponding to higher penetration depths is fit the calculated elastic modulus is determined to be $2.8 \mathrm{GPa}$, which corresponds well with standard values of PS elastic modulus. The primary stress from the applied force from the tip onto the surface extends farther into the subsurface as the tip radius increases for a given penetration depth [36]. Therefore, in the case the large tip radius probe is able to better probe the bulk material away beneath the compliant surface. Facilitated by a simple fitting algorithm, this routine could perhaps be very useful in exploring different mechanical responses with penetration depth.

Further examples of the usefulness of varying tip size when making micromechanical measurements of soft materials is the ability to switch between strictly surface modulus (with a depth of 10 s of $n m$ ) while using small radii probes, to examining the structural rigidity of a sample of with a large tip radii. There are several examples in the literature in which the structural rigidity of small cells is measured by examining their mechanical properties with large colloidal probes.

\section{Conclusions}

As AFM-based force spectroscopy takes an even more prominent role in determining the micromechanical properties of soft materials, biomaterials, and nanocomposites the precision of data analysis routines should also be refined. Here we have described in detail several contact mechanics models for commonly encountered tip geometries: paraboloidal, conical, and spherical, and used a variety of soft materials to exemplify situations in which each model should be employed. Additionally we have demonstrated the usefulness of our home-built analysis program, MMA Software 
(licensed freeware package available from authors), in calculating micromechanical properties of soft materials from FDC data.

In this paper, we showed how quickly results could deviate after simple incorrect assumption of tip geometry, and use of the equations of a paraboloidal punch rather than those for a spherical punch for FDC analysis. FDCs collected on the soft elastic surface of a commercial contact lens were observed to exhibit behavior that is not fitted very well with the parabolic model. As a result, we suggest that in some cases the sharp tip of a brand new probe could actually resemble more a hemispherically-capped cylindrical punch with a finite limit to contact radius than a paraboloidal punch which has a contact radius that can continuously increase. Unfortunately, there is no analytical solution to the spherical equations and numerical analysis can be very cumbersome. However we have described our simple algorithm for working with spherical equations, which will be practical for real-life applications.

Analysis routines for utilizing the conical model here showed that the initial portions of FDCs could be used with the parabolic model, but as indentation depth grows, the blunt tip apex plays a decreasing role in the overall contact area. Thus calculations using the initial indentation with the parabolic model and the latter stages of indentation with the conical model gave results within on standard deviation. However, the caveat to this approach is that a significant amount of data points need to be collected (i.e. high enough indentation depths need to be achieved) in order to reduce the effect of the blunted tip apex for the conical model to fit well.

Finally the effect of tip radius can be briefly discussed. In general, it is well known that larger probes facilitate mechanical properties determination while reducing the probability of plastic deformation. We have also shown some specific examples when a sharp probe could be preferential to a dull probe, especially in the case where precise positioning is required for the determination of the properties of extremely fine surface features. In the case of a large radius probe, the stress distribution immediately below the tip-sample contact point extends farther into the subsurface. As a result, the FDC behavior observed on the same material surface may be different when probed with 
different radii probes. By probing farther into the bulk using a large radius probe, we could detect a modulus which more closely matched literature values.

\section{Acknowledgements}

We are grateful for the financial support from the National Science Foundation, DMR1505234 and CBET-1402712 projects; Air Force Office of Scientific Research, FA955014-1-0269 award, as well as funding received from the Microanalysis Center at the Georgia Institute of Technology. 


\section{Figures}

A
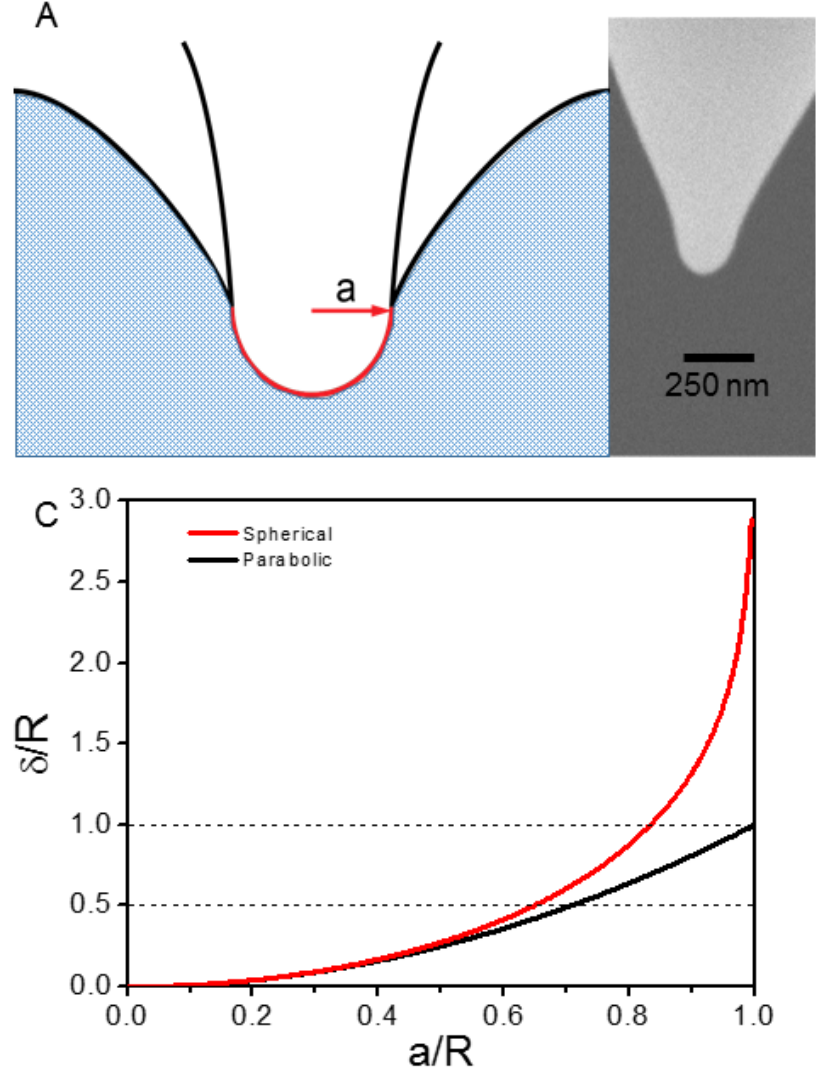

B
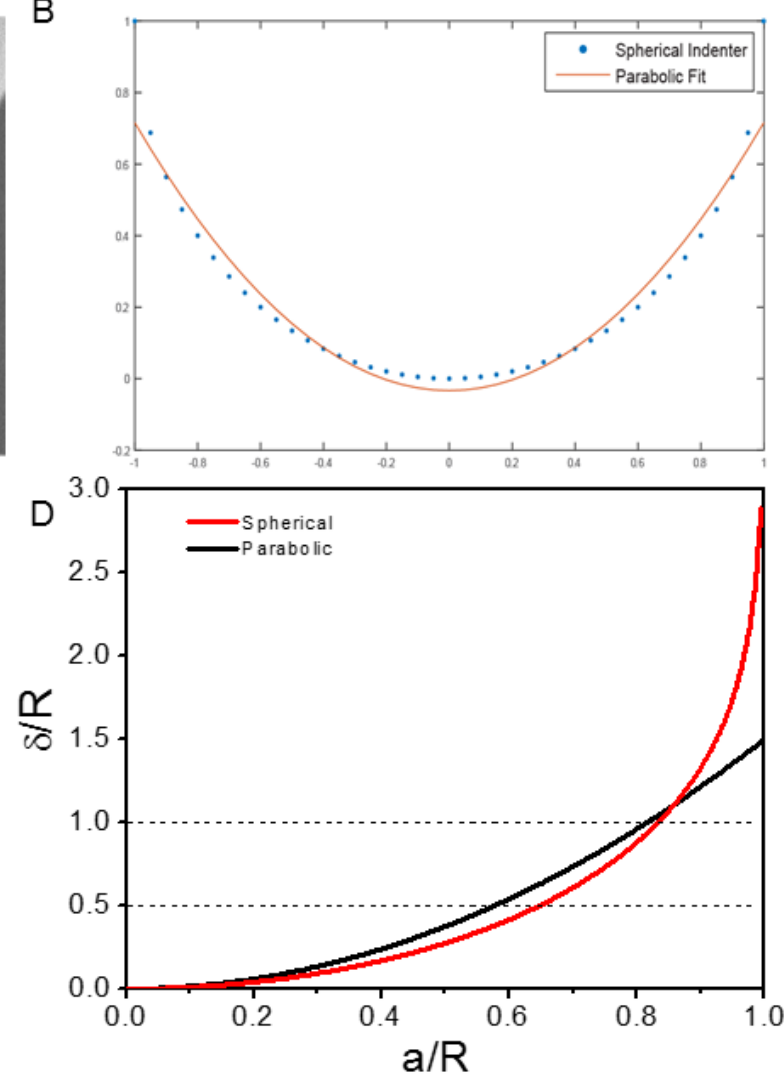

Figure 1: (A) An example of how the end of an AFM tip could behave as a spherical indenter wherein the maximum contact radius does not exceed the radius of the spherical cap. Also shown is an SEM of an annealed AFM tip which displays a similar shape to the schematic. (B) Example of fitting of the spherical indenter with the parabolic models. (C) Differences between the contact area changes with penetration for the spherical indenter with radius $\mathrm{R}$ and parabolic indenter with the apex curvature 1/R. (D) Comparison between parabolic and spherical models with radius of curvature for the apex of parabola equals to $0.6675 \mathrm{R}$. 

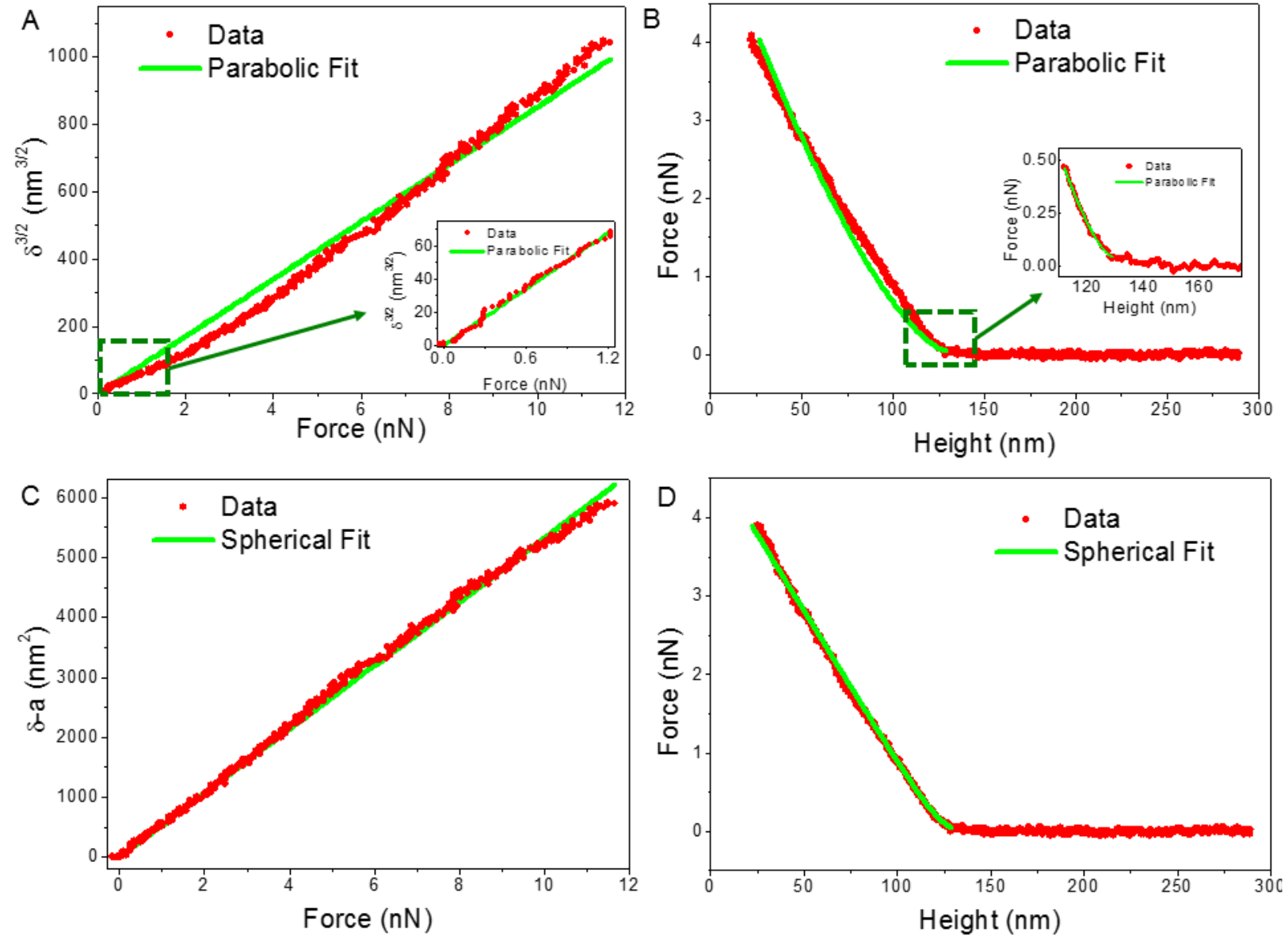

Figure 2: Force distance curves taken on Balafilcon $A$ contact lens surfaces in fluid and fit with either Sneddon's model for a rigid paraboloidal punch ( $A$ and $B$ ) or for a rigid spherical punch ( $C$ and $D)$. Inset in $A$ and $B$ are initial regions of the FDC which have been fitted separately with the parabolic model, exhibiting how well only the very first portion of indentation in this case can be considered parabolic. 

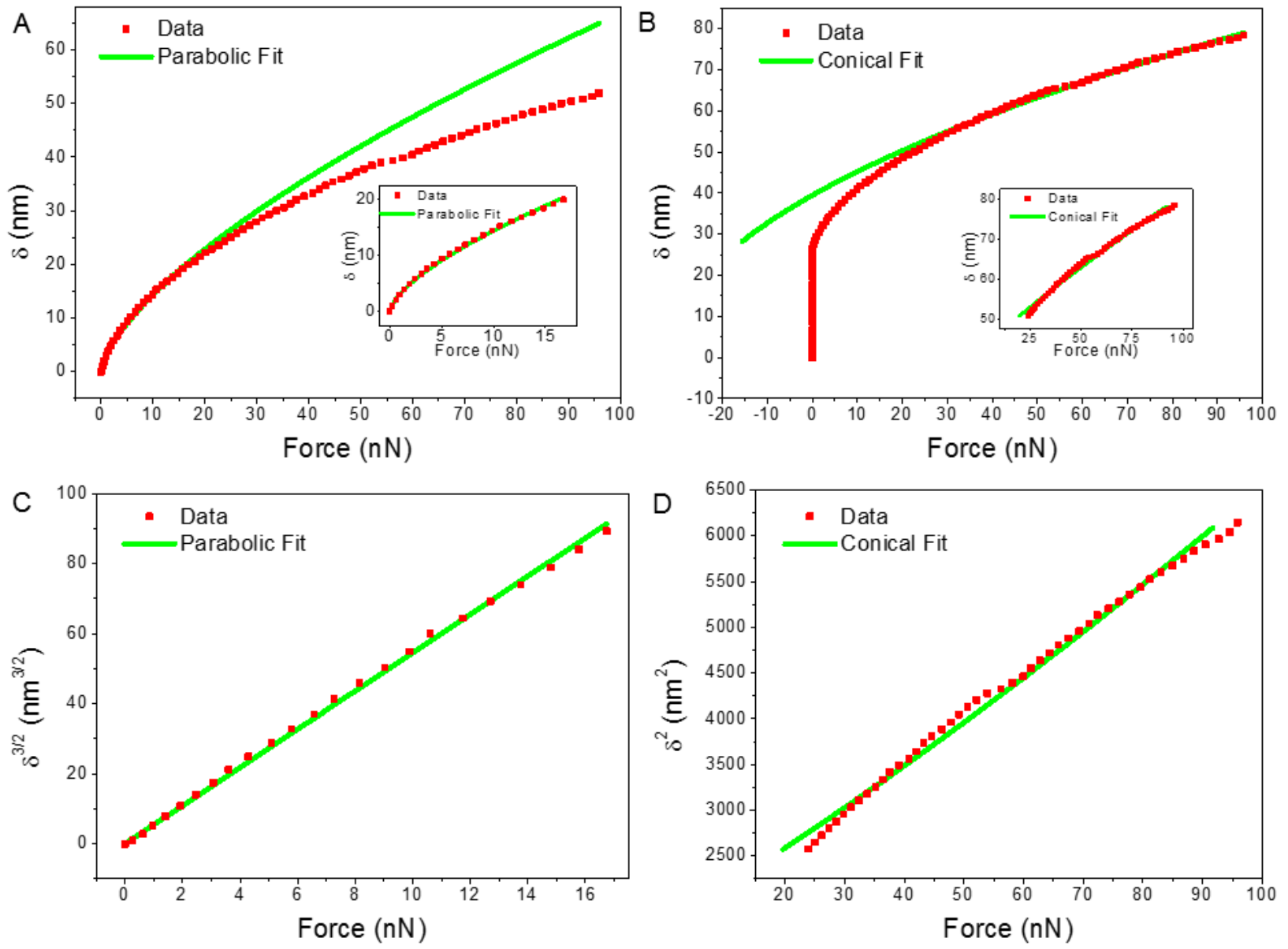

Figure 3: A comparison of FDC fitting using the equations for a parabolic punch (A \& C) and the equations for a conical punch in our silk ionomer system (B \& D). The top row shows the fit of our model function to the whole dataset, while the inset shows the fit in a smaller region more appropriately suited for the specific model (i.e. early in the FDC for parabolic model, and later in the FDC for conical model). Note that the second row is the fitted region plotted as $\delta^{3 / 2}$ or $\delta^{2}$ to show linearity. 

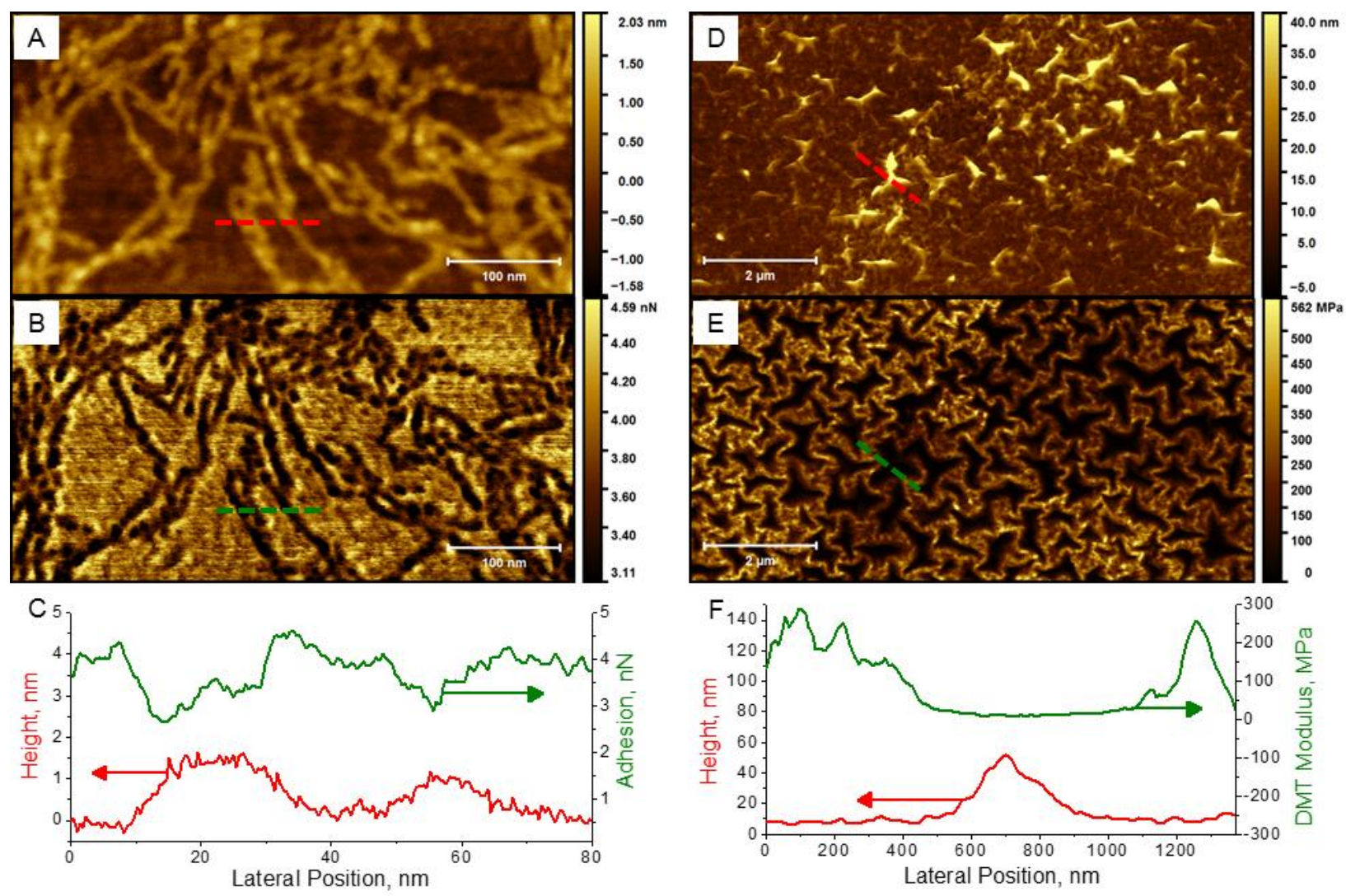

Figure 4: (A) Height image of nanofibrils of regenerated silk fibroin biomacromolecules cast on a silicon wafer. (B) Surface adhesion collected simultaneously, showing a distinct difference between the silk and the silicon ssurface. (C) Sectional profiles of height and adhesion data taken from the locations in $\mathrm{A}$ and $\mathrm{C}$ denoted by dashed lines. (D) Topography of silk ionomer surface with raised regions occuring as the result of buckling. (E) DMT modulus image acquired simultaneously which highlights the need for a sharp probe as the unsupported buckled regions give unrealisticly low values of modulus while the supported regions can be used for accurate estimation of mechanical properties. (F) Sectional profiles of height and adhesion data taken from the locations in $\mathrm{D}$ and $\mathrm{E}$ denoted by dashed lines. 

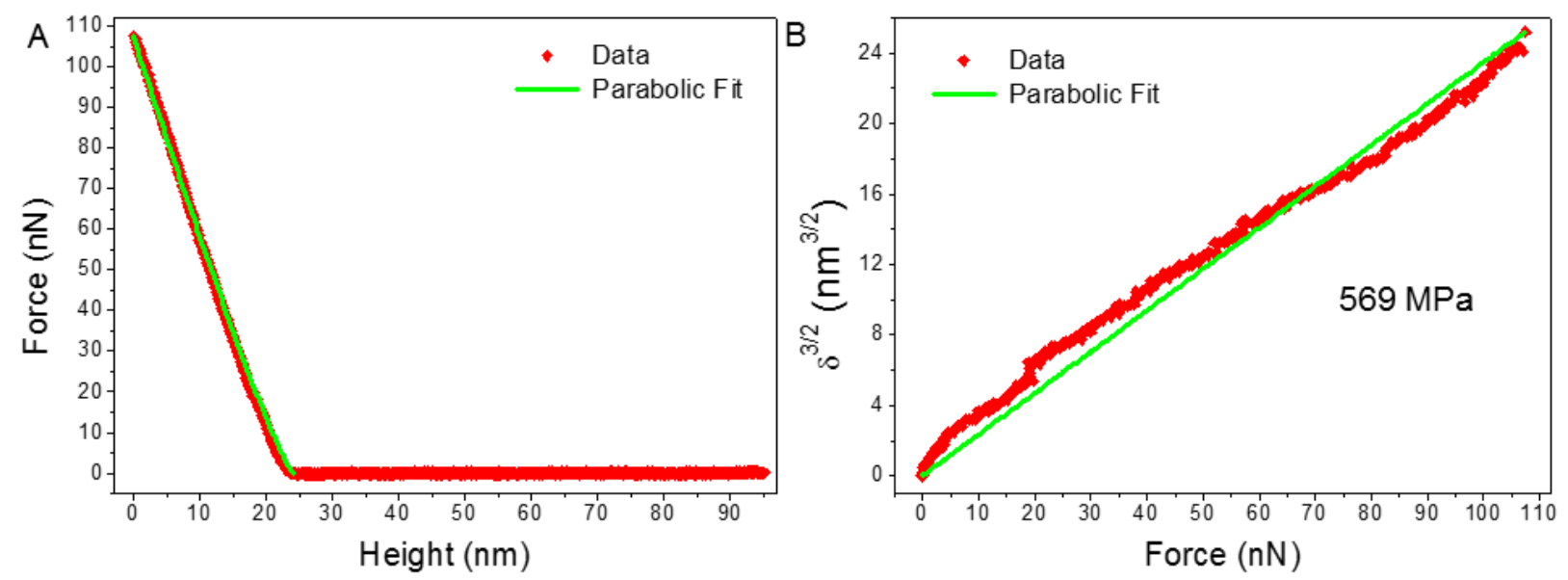

Figure 5: (A) Force-distance curve taken with a sharp probe (radius of curvature 20 $\mathrm{nm}$ ) on a cast polystyrene substrate with the maximum sample penetration depth of 9 nm. (B) $\delta^{3 / 2}$ versus applied force used for fitting with Sneddon's model for a rigid parabolic punch. In this case, the elastic modulus was calculated to be $569 \mathrm{MPa}$. 

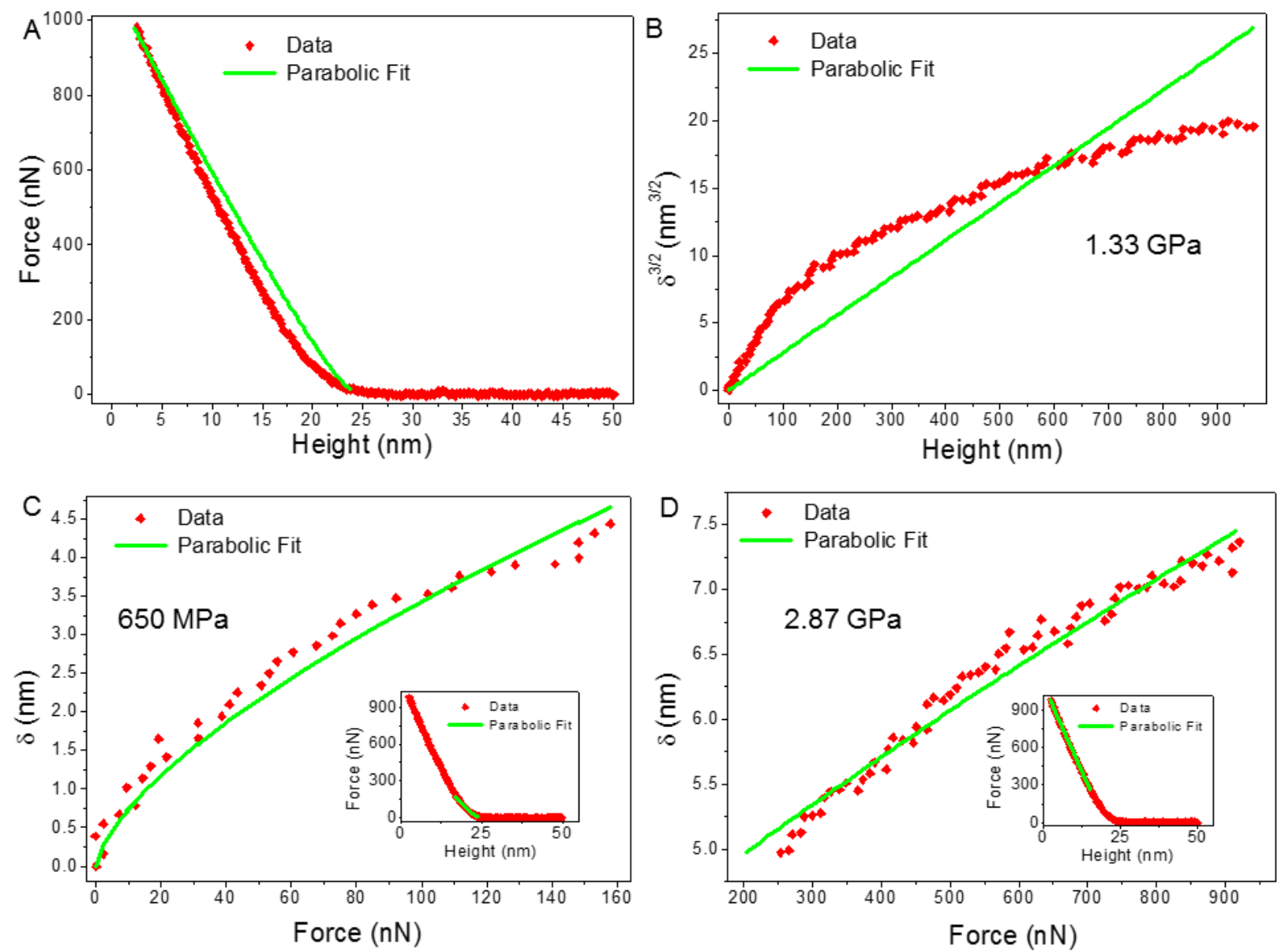

Figure 6: (A) Force-distance curve taken with a large, annealed probe (radius of curvature $\sim 330 \mathrm{~nm}$ ) on the same cast polystyrene substrate as the measurements in Fig. 6. In this measurement the maximum sample penetration depth is $8 \mathrm{~nm}$. (B) $\delta^{3 / 2}$ versus applied force used for fitting with Sneddon's model for a rigid parabolic punch with the calculated elastic modulus of approximately $1.3 \mathrm{GPa}$. The bottom two panels show the fitting of $\delta$ (used here to show actual deformation levels) versus applied force for initial penetration $(C)$ and final penetration depths $(D)$. Inset is the original FDC and fitting for the corresponding portion of the curve. 


\section{References}

[1] Kovalev A, Shulha H, Lemieux M, Myshkin N, and Tsukruk VV. J Mater Res 2004;19(03):716-728.

[2] Chyasnavichyus M, Young SL, and Tsukruk VV. Jpn. J. Appl. Phys.2015;54(8S2):08LA02.

[3] Seidlits SK, Khaing ZZ, Petersen RR, Nickels JD, Vanscoy JE, Shear JB, and Schmidt CE. Biomaterials 2010;31(14):3930-3940.

[4] Tsukruk VV and Singamaneni S. Scanning Probe Microscopy of Soft Matter: Fundamentals and Practices. 2012: Wiley, Weinheim.

[5] Müller DJ and Dufrêne YF. Trends Cell Biol 2011;21(8):461-469.

[6] Iwamoto S, Kai W, Isogai A, and Iwata T. Biomacromolecules 2009;10(9):2571-2576.

[7] Young TJ, Monclus MA, Burnett TL, Broughton WR, Ogin SL, and Smith PA. Meas Sci Technol 2011;22(12):125703.

[8] Häberle W, Hörber JKH, and Binnig G. J. Vac. Sci. Technol. B 1991;9(2):1210-1213.

[9] Cappella B and Kaliappan SK. Macromolecules 2006;39(26):9243-9252.

[10] Tsui OKC, Wang XP, Ho JYL, Ng TK, and Xiao X. Macromolecules 2000;33(11):41984204.

[11] Tsukruk VV, Gorbunov VV, Huang Z, and Chizhik SA. Polym Int 2000;49(5):441-444.

[12] McConney ME, Singamaneni S, and Tsukruk VV. Polym Rev 2010;50(3):235-286.

[13] Chizhik SA, Huang Z, Gorbunov VV, Myshkin NK, and Tsukruk VV. Langmuir 1998;14(10):2606-2609.

[14] Butt H-J, Cappella B, and Kappl M. Surf Sci Rep 2005;59(1-6):1-152.

[15] Chyasnavichyus M, Young SL, and Tsukruk VV. Polymer 2014;55(23):6091-6101

[16] Young SL, Chyasnavichyus M, Erko M, Barth FG, Fratzl P, Zlotnikov I, Politi Y, and Tsukruk VV. Acta Biomater 2014;10(11):4832-4842.

[17] Lisunova MO, Drachuk I, Shchepelina OA, Anderson KD, and Tsukruk VV. Langmuir 2011;27(17):11157-11165.

[18] Murphy AR, John PS, and Kaplan DL. Biomaterials 2008;29(19):2829-2838.

[19] Gupta MK, Singamaneni S, McConney M, Drummy LF, Naik RR, and Tsukruk VV. Adv Mater 2010;22(1):115-119.

[20] Young SL, Gupta M, Hanske C, Fery A, Scheibel T, and Tsukruk VV. Biomacromolecules 2012;13(10):3189-3199.

[21] Drachuk I, Calabrese R, Harbaugh S, Kelley-Loughnane N, Kaplan DL, Stone M, and Tsukruk VV. ACS Nano 2015;9(2):1219-1235.

[22] Tian F, Qian X, and Villarrubia JS. Ultramicroscopy 2008;109(1):44-53.

[23] Levy RL and Maaloum M. Nanotechnology 2002;13(1):33

[24] Cook SM, Schaffer TE, Chynoweth KM, Wigton M, Simmonds RW, and Lang KM. Nanotechnology 2006;17(9):2135.

[25] Benıtez R, Moreno-Flores S, Bolós VJ, Toca-Herrera JL Microsc. Res Tech 2013;76(8): 870-876.

[26] Johnson KL. Greenwood, JA J Colloid Interface Sci 1997;192: 326-333.

[27] Zhen, G. and Dobrynin. AV Langmuir 2015;31(45):12520-12529.

[28] Chyasnavichyus M, Young SL, and Tsukruk VV. Langmuir 2014;30(35):10566-10582.

[29] Cappella B, Kaliappan SK, and Sturm H. Macromolecules 2005;38(5):1874-1881. 
[30] Sneddon IN. Int J Eng Sci 1965;3(1):47-57.

[31] Belikov S, Magonov S, Erina N, Huang L, Su C, Rice A, Meyer C et al. J Phys: Conf Ser 2007;61(1):1303-1307

[32] Kopycinska-Müller M, Geiss RH, and Hurley DC. Ultramicroscopy 2006;106(6):466-474.

[33] Flater EE, Zacharakis-Jutz GE, Dumba BG, White IA, and Clifford CA. Ultramicroscopy 2014;146:130-143.

[34] Berla LA, Allen AM, Han SM, and Nix WD. J Mater Res 2010;25(04):735-745.

[35] Oliver WC and Pharr GM. J Mater Res 2004;19(01):3-20.

[36] Johnson KL. Contact Mechanics. 1985: Cambridge University Press, Cambridge. 